\title{
Processo de morte para as mulheres que vivenciam o aborto sob a perspectiva da enfermagem
}

Death process for women who experience abortion from a nursing perspective

Proceso de muerte de mujeres que experimentan el aborto desde la perspectiva de la enfermería

Recebido: 12/09/2021 | Revisado: 20/09/2021 | Aceito: 02/10/2021 | Publicado: 03/10/2021

Luanny Regina de Oliveira Santos

ORCID: https://orcid.org/0000-0002-5017-6974

Universidade Federal Fluminense, Brasil

E-mail: luanny.enf09@gmail.com

Ana Clara Dias da Silva

ORCID: https://orcid.org/0000-0002-1498-8545

Universidade Iguaçu, Brasil

E-mail: anaclaradiass99@hotmail.com

Ana Karolainny da Silva Barbosa

ORCID: https://orcid.org/0000-0002-6957-3515

Universidade de Pernambuco, Brasil

E-mail: anakaarolainny@gmail.com

Anna Flávia Pereira Moutinho da Silva ORCID: https://orcid.org/0000-0002-0050-9064 Universidade Estácio de Sá, Brasil

E-mail: anna.fla.12@gmail.com

Brenda Giovanna Rufino Ferreira de Almeida ORCID: https://orcid.org/0000-0002-5665-7541 Universidade Estácio de Sá, Brasil

E-mail: brendagiovanna@hotmail.com

Dayana Campos de Oliveira Cardoso ORCID: https://orcid.org/0000-0002-5031-6041 Universidade Estácio de Sá, Brasil

E-mail: Camposdayana2015@hotmail.com

Elziane Alves Ribeiro

ORCID: https://orcid.org/0000-0001-5366-2460

Universidade Estácio de Sá, Brasil

E-mail: elzialvesribeiro@gmail.com Jéssica Silva de Santana

ORCID: https://orcid.org/0000-0002-3470-778X

Universidade Estácio de Sá, Brasil

E-mail: jsantanaa.estacio@gmail.com

Jonathan Lima Melhardo

ORCID: https://orcid.org/0000-0003-1881-8866

Universidade do Grande Rio, Brasil

E-mail: j.melhardo01@gmail.com

Jullyan Sttefanny Martins Castelo Branco

ORCID: https://orcid.org/0000-0001-7734-116X

Cento Universitário Santo Agostinho, Brasil

E-mail: juhcastelobranco@gmail.com

Laide dos Santos Brasil Silva

ORCID: https://orcid.org/0000-0001-5593-7173

Cento Universitário Santo Agostinho, Brasil E-mail: Laidebr.98@gmail.com

Larissa Carvalho Teixeira

ORCID: https://orcid.org/0000-0002-4439-7578

Universidade Estácio de Sá, Brasil

E-mail: larissacarvalho.tjc@gmail.com

Larissa Lessa dos Santos

ORCID: https://orcid.org/0000-0002-8947-6691

Universidade Estácio de Sá, Brasil

E-mail: larissalessaa@hotmail.com

Larissa Salles de Souza

ORCID: https://orcid.org/0000-0002-8202-7834

Universidade Estácio de Sá, Brasil

E-mail: larisalles2735@gmail.com 


\author{
Marah Christini Rodrigues de Sousa \\ ORCID: https://orcid.org/0000-0002-1094-7754 \\ Cento Universitário Santo Agostinho, Brasil \\ E-mail: marahchristini@gmail.com \\ Maria Deluany Guilherme Duarte \\ ORCID: https://orcid.org/ 0000-0002-5631-5349 \\ Universidade Paulista, Brasil \\ E-mail: maria_dgd@hotmail.com \\ Mariana Lopes Teixeira \\ ORCID: https://orcid.org/0000-0002-1382-3271 \\ Universidade Estácio de Sá, Brasil \\ E-mail: marianalopestx@gmail.com \\ Stéphanie da Silva Pereira \\ ORCID: https://orcid.org/0000-0002-2952-5914 \\ Universidade Estácio de Sá, Brasil \\ E-mail: pereira.stephanie1999@gmail.com \\ Thaís Pessanha Moreira \\ ORCID: https://orcid.org/0000-0003-2794-6536 \\ Universidade Estácio de Sá, Brasil \\ E-mail: thaispessanha@icloud.com \\ Paula de Carvalho Pereira Pitombeira \\ ORCID: https://orcid.org/0000-0002-7316-0713 \\ Universidade Federal do Estado do Rio de Janeiro, Brasil \\ E-mail: paulapitombeira.pp@gmail.com
}

\title{
Resumo
}

Introdução: Aborto é a interrupção da gestação de forma espontânea ou provocada. Considerara-se um tema delicado por envolver questões culturais, religiosas, legais e morais. Justificativa: Há poucos estudos acadêmicos sobre a temática, o que torna a pesquisa imprescindível. Objetivo: Compreender o processo de morte no entendimento das mulheres que vivenciam o aborto e analisar a assistência da enfermagem às consequências ocasionadas. Metodologia: Revisão integrativa descritiva e qualitativa. Os critérios de inclusão foram: artigos completos, em língua portuguesa, recorte temporal de 10 anos. Os critérios de exclusão foram: artigos duplicados e que desviam do tema proposto. Foram selecionados 05 artigos para leitura e realização da pesquisa. Discussão: Restrições econômica, objetivos pessoais, não aceitação familiar, ausência de parceiro e violência doméstica podem levar ao aborto. Estudos revelam que mulheres pardas ou negras tiveram maior índice nos abortos induzidos do que mulheres brancas. A falta de compreensão e o julgamento fazem com que essas mulheres não encontrem uma forma de traduzir os sentimentos e superar a perda, podendo levá-las à uma vida de autopunição, risco de depressão e vícios, devido ao trauma da experiência. $\mathrm{O}$ enfermeiro, por ser quem tem o primeiro contato com a paciente, pode sugerir que seja encaminhada ao psicólogo para que tenha o acompanhamento necessário. Conclusão: Preservar a vida da mulher assim como a saúde mental é uma questão de saúde pública que dependerá diretamente do suporte que iremos dispor, independente de questões pessoais, a assistência deverá ser integral, universal e visando sempre os princípios de humanização.

Palavras-chave: Morte; Aborto; Mulher.

\begin{abstract}
Introduction: Abortion is the interruption of pregnancy spontaneously or induced. It is considered a delicate topic for embrace cultural, religious, legal, and moral issues. Justification: There are few academic studies on the subject, which makes the research essential. Objective: To understand the process of death through the eyes of women who experience abortion and analyze nursing care for the caused consequences. Methodology: Descriptive and qualitative integrative review. Inclusion criteria were: complete articles, in Portuguese, with a 10-year time frame. Exclusion criteria were: duplicated articles that deviate from the proposed theme. 05 articles were selected for reading and conducting the research. Discussion: Economic restrictions, personal goals, family non-acceptance, absence of a partner, and domestic violence can lead to abortion. Studies reveal that brown or black women had a high rate of induced abortions than white women. The lack of information and judgment makes these women not find a way to interpret their feelings and overcome the loss, which can lead them to a life of self-punishment, risk of depression and addictions, due to the trauma of the experience. The nurse, being the one who has the first contact with the patient, can suggest that she be referred to the psychologist so that she can have the necessary follow-up. Conclusion: Preserve women's lives as well as mental health is a public health issue that will depend directly on the support we will give, regardless of personal issues, care should be complete, universal, and always aiming at the principles of humanization.
\end{abstract} Keywords: Death; Abortion; Woman.

\section{Resumen}

Introducción: El aborto es la interrupción del embarazo de forma espontánea o inducida. Se ha considerado un tema delicado porque involucra cuestiones culturales, religiosas, legales y morales. Justificación: Existen pocos estudios académicos sobre el tema, lo que hace imprescindible la investigación. Objetivo: Comprender el proceso de la muerte 
en la comprensión de las mujeres que experimentan el aborto y analizar los cuidados de enfermería por las consecuencias que ocasiona. Metodología: Revisión integrativa descriptiva y cualitativa. Los criterios de inclusión fueron: artículos completos, en portugués, con un plazo de 10 años. Los criterios de exclusión fueron: artículos duplicados que se desvían del tema propuesto. Se seleccionaron 05 artículos para la lectura y realización de la investigación. Discusión: Las restricciones económicas, los objetivos personales, la no aceptación familiar, la falta de pareja y la violencia doméstica pueden llevar al aborto. Los estudios revelan que las mujeres morenas o negras tenían una tasa más alta de abortos inducidos que las mujeres blancas. La falta de comprensión y juicio hace que estas mujeres no encuentren la forma de plasmar sus sentimientos y superar la pérdida, lo que puede llevarlas a una vida de autocastigo, riesgo de depresión y adicciones, debido al trauma de la experiencia. La enfermera, al ser quien tiene el primer contacto con la paciente, puede sugerirle que sea referida al psicólogo para que tenga el seguimiento necesario. Conclusión: Preservar la vida de las mujeres así como la salud mental es un tema de salud pública que dependerá directamente del apoyo que tengamos, independientemente de las cuestiones personales, la atención debe ser integral, universal y siempre apuntando a los principios de la humanización.

Palabras clave: Muerte; Aborto; Mujer.

\section{Introdução}

Atualmente o aborto é considerado um tema crítico, pois se trata de uma temática que abrange questões culturais, religiosas, legais e morais. Entretanto, historicamente é possível verificar que o aborto é inserido em distintas civilizações com significados diversos.

Na Grécia e na Roma antiga, utilizavam o aborto como forma de limitar o crescimento populacional. O controle de natalidade nas cidades era visto como questão econômica. Com o advento do cristianismo, passou-se a condenar o aborto como base no mandamento "não matarás", os imperadores e governantes da época decretaram então o aborto como homicídio. No século XVIII, a Revolução Francesa reforça o banimento do aborto, pois considerava o feto como um futuro trabalhador e soldado (Borsari et al., 2012).

Posteriormente, no século XX, países emergentes adotaram a legalidade do aborto, para garantir a saúde das trabalhadoras e controlar uma possível crise econômica. Enquanto a queda populacional em outros países por consequência da Primeira Guerra Mundial o aborto foi proibido como forma de aumentar a população da região afetada (Galeotti, 2007).

No Brasil, o aborto induzido é descrito desde a colonização, onde indígenas praticavam o aborto pois sofriam com a dissolução de suas famílias, e condições de miséria e violência. As práticas abortivas no Brasil colonial variavam desde chás até a introdução de objetos cortantes. Era comum ocorrer a morte dessas mulheres." De acordo com Del Priore, “ao tentar livrar-se do fruto indesejado, as mães acabavam por matar-se. O consumo de chás e poções abortivas acabava por envenená-las (Del Priori, 1993).

A partir da década de 60 o surgimento e crescimento da revolução sexual e dos ideais feministas deram origem a uma série de discussões sobre a legalidade do aborto. Com a realização de estudos em saúde pública, o aborto passou a ser problematizado como fato social e não mais como desvio moral, onde se percebeu a importância da sua legitimidade para a garantia da saúde da mulher (Denzin et al., 2000).

Neste contexto, começa a surgir ênfase em compreender o processo de morte no entendimento da mulher, seja a perda causada por aborto induzido ou espontâneo.

Diante do exposto, o estudo apresenta como objeto de pesquisa, o processo de morte vivenciado por mulheres que sofreram aborto.

O estudo apresenta como questão norteadora: Como ocorre o processo de morte no entendimento das mulheres que vivenciam o aborto, seja ele induzido ou natural?

Para tentar responder essa pergunta, surgiram os seguintes objetivos de pesquisa: Compreender o processo de morte no entendimento das mulheres que vivenciam o aborto e analisar a assistência da enfermagem às consequências físicas e mentais ocasionadas. 


\subsection{Justificativa}

Esse estudo se justifica a partir da dificuldade dos acadêmicos de enfermagem, da sociedade e dos profissionais de saúde ao debater sobre a temática, engloba aspectos políticos e pré-conceitos sociais, visando buscar melhoria no desempenho e no conhecimento o que acarretará na evolução do domínio destes âmbitos para dissertar e entender melhor o assunto. Através disto foi possível analisar que há pouco estudo acadêmico sobre o tema discutido, tornando imprescindível a pesquisa cientifica nesta área.

\subsection{Relevância}

A contribuição do estudo para o meio acadêmico e enfermeiros ingressantes se faz com a intenção de informar e buscar reflexão sobre a temática, pois será de grande importância tendo em vista que irão realizar esta assistência futuramente. Para os profissionais atuantes na área a relevância é para auxiliar na atualização e aprimoramento na assistência, garantindo um atendimento amplo e humanizado, atendendo a todas as necessidades da paciente. A relevância social visa levar a sociedade o conhecimento científico que geralmente não os é oferecido, já que poderão fazer uso dos dados reunidos nesta pesquisa.

\section{Metodologia}

Trata-se de um estudo de abordagem qualitativa, descritiva, do tipo revisão integrativa, onde visa compreender o processo de morte para as mulheres que vivenciam o aborto sob a perspectiva da enfermagem.

A pesquisa qualitativa pode ser definida como a que se fundamenta principalmente em análises qualitativas, caracterizando-se, em princípio, pela não utilização de instrumental estatístico na análise dos dados (Souza, 2007).

Deste modo, não é apenas a "pesquisa não quantitativa", tendo desenvolvido sua própria identidade. Assim, visa entender, descrever e explicar os fenômenos sociais de modos diferentes, através da análise de experiências individuais e grupais, exame de interações e comunicações que estejam se desenvolvendo, assim como da investigação de documentos (textos, imagens, filmes ou músicas) ou traços semelhantes de experiências e integrações (Flick, 2009).

Para a construção do artigo abordou-se a revisão qualitativa que é a denominação para uma "complexa e interconectada família de termos, conceitos e hipóteses", provenientes de várias disciplinas e áreas de conhecimento. Consiste num cenário de práticas interpretativas que torna o mundo visível e estas transformam o mundo, tornando o mundo uma série de representações, incluindo notas de campo, entrevistas, diálogos, fotografias, gravações e memórias pessoais (Denzin et al. 2000)

A pesquisa descritiva exige do investigador uma série de informações sobre o que deseja pesquisar. Esse tipo de estudo pretende descrever os fatos e fenômenos de determinada realidade (Alves, 2013).

A estratégia de identificação e seleção dos artigos originou-se através da busca da Biblioteca Virtual de Saúde (BVS), no mês de março de 2020. Inicialmente a busca de materiais que contivessem em seu resumo os descritores em ciências da saúde (DeCS): Morte, Aborto e Mulher. Na qual emergiram, 385 artigos emergiram usando tais descritores.

Foi realizado um recorte temporal dos últimos 10 anos (2009-2019), devido à maior necessidade de discussão a respeito da temática. Como critérios de inclusão, foram adotados documentos com texto completo e idioma em língua portuguesa. Os critérios de exclusão foram os estudos que não atendessem aos critérios citados anteriormente e artigos duplicados.

Dos 385 artigos emergidos, apenas 13 artigos foram encontrados após adicionar os filtros, dos quais 4 contribuíram para a realização da pesquisa com os critérios de inclusão, os quais procederam-se à leitura minuciosa para a construção do conteúdo, destacando aqueles que responderam ao objetivo proposto pelo estudo, com intuito de organizar os dados de acordo 
com o objetivo proposto.

Os artigos foram tabelados por título, autores, periódico, objetivos, resultados e conclusão. A partir de então iniciou a análise destes que foram agrupados por similaridade sob forma de categorias.

\section{Resultados}

Os artigos foram tabelados e obtido as seguintes informações da pesquisa: título, autores, país de origem, periódico, base de dados, qualis, objetivos, método e nível de evidência (Tabela 1).

Tabela 1. Características dos artigos analisados no período de 2009 a 2019 na base de dados da Biblioteca Virtual de Saúde (BVS)

\begin{tabular}{|c|c|c|c|c|c|c|c|}
\hline Título & Autores & $\begin{array}{l}\text { País de } \\
\text { origem }\end{array}$ & $\begin{array}{c}\text { Periódico e } \\
\text { base de dados }\end{array}$ & Qualis & Objetivo & Método & $\begin{array}{l}\text { Nível de } \\
\text { evidência }\end{array}$ \\
\hline $\begin{array}{l}\text { Aborto } \\
\text { provocado: } \\
\text { representações } \\
\text { sociais de } \\
\text { mulheres. }\end{array}$ & $\begin{array}{l}\text { Bárbara Angélica } \\
\text { Gómez Pérez; } \\
\text { Nadirlene } \\
\text { Pereira Gomes; Maria } \\
\text { de Fátima de Souza } \\
\text { Santos; Normelélia } \\
\text { Maria Freire Diniz. }\end{array}$ & Brasil & $\begin{array}{l}\text { Rev. } \\
\text { enferm. } \\
\text { UERJ/ } \\
\text { LILACS, } \\
\text { BDENF }\end{array}$ & A4 & $\begin{array}{l}\text { Aprender o conteúdo e } \\
\text { a estrutura das } \\
\text { representações sociais } \\
\text { de mulheres sobre o } \\
\text { aborto provocado. }\end{array}$ & $\begin{array}{l}\text { Estudo } \\
\text { descritivo } \\
\text { com } \\
\text { abordagem } \\
\text { qualitativa. }\end{array}$ & IV \\
\hline $\begin{array}{lr}\text { O } & \text { aborto } \\
\text { inseguro é um } \\
\text { problema de } \\
\text { saúde pública. }\end{array}$ & $\begin{array}{lr}\text { Cristina } & \text { Mendes } \\
\text { Gigliotti } & \text { Borsari; } \\
\text { Roseli } & \text { Mieko } \\
\text { Yamamoto } & \text { Nomura; } \\
\text { Gláucia } & \text { Guerra } \\
\text { Benute; } & \text { Danielle } \\
\text { Nonnenmacher; Mara } \\
\text { Cristina } & \text { Souza de } \\
\text { Lucia; } & \text { Rossana } \\
\text { Pulcineli } & \text { Vieira } \\
\text { Francisco. } & \\
\end{array}$ & Brasil & $\begin{array}{l}\text { Feminina/ } \\
\text { LILACS }\end{array}$ & B3 & $\begin{array}{l}\text { Analisar as pesquisas } \\
\text { que abordam o aborto } \\
\text { provocado ou inseguro } \\
\text { no Brasil. }\end{array}$ & $\begin{array}{l}\text { Estudo } \\
\text { descritivo } \\
\text { com } \\
\text { abordagem } \\
\text { qualitativa. }\end{array}$ & III \\
\hline $\begin{array}{ll}\text { Causas } & \\
\text { Múltiplas de } \\
\text { mortalidade } \\
\text { materna } \\
\text { relacionado ao } \\
\text { aborto no } \\
\text { Estado de Minas } \\
\text { Gerais } \\
\end{array}$ & $\begin{array}{l}\text { Eunice } \\
\text { Martins; Prancisca } \\
\text { Ferraz Bolyanna } \\
\text { Almeida;Cilene de de } \\
\text { Oliveira Paixão; Paula } \\
\text { Gonçalves Bicalho; } \\
\text { Lívia de Souza; } \\
\text { Pancrácio de Enrico. }\end{array}$ & Brasil & $\begin{array}{ll}\text { Cad. } & \text { De } \\
\text { Saúde } & \\
\text { Pública/ } & \\
\text { LILACS } & \end{array}$ & B3 & $\begin{array}{l}\text { Analisar a evolução da } \\
\text { mortalidade materna } \\
\text { por aborto em Minas } \\
\text { Gerais, no período de } \\
2000 \text { a 2011, sob } \\
\text { enfoque das causas } \\
\text { múltiplas da morte. }\end{array}$ & $\begin{array}{l}\text { Estudo } \\
\text { descritivo } \\
\text { com } \\
\text { abordagem } \\
\text { qualitativa. }\end{array}$ & III \\
\hline $\begin{array}{lr}\text { O } & \text { aborto } \\
\text { vivenciado } & \text { em } \\
\text { situação } & \text { de } \\
\text { anencefalia } & \end{array}$ & Massud; Munir. & Brasil & & B4 & $\begin{array}{l}\text { A anencefalia, um grave } \\
\text { defeito embrionário do } \\
\text { sistema nervoso central, } \\
\text { tem sido alvo de } \\
\text { acalorados debates em } \\
\text { diversos setores da } \\
\text { sociedade brasileira. O } \\
\text { tema suscita grande } \\
\text { interesse ao envolver a } \\
\text { questão do abortamento. } \\
\text { Ademais, novamente os } \\
\text { direitos femininos são } \\
\text { também trazidos à baila, } \\
\text { em face da } \\
\text { possibilidade de se } \\
\text { conceder à mulher a } \\
\text { decisão de continuar ou } \\
\text { não a gestar feto } \\
\text { anencéfalo. }\end{array}$ & & IV \\
\hline
\end{tabular}

Fonte: Autores. 


\section{Discussão}

Após a leitura minuciosa dos artigos selecionados, emergiram as seguintes categorias: Consequências do aborto no entendimento da mulher; Atuação da enfermagem frente ao processo de morte vivenciado por mulheres que sofreram aborto.

O aborto é permitido somente em algumas circunstâncias no Brasil, como estupro, quando há risco de vida para a mulher e em 2012 o Supremos Tribunal Federal (STF) incorporou a gravidez de feto com anencefalia como possibilidade de interrupção da gravidez. Porém, desde a década de 70 as mulheres vêm questionando mais as desigualdades e discutem temas como direitos ao seu corpo, cabendo assim a tomada de decisão de realizar o aborto ser única e exclusivamente da mulher.

Identifica-se ao longo da história que as motivações que levam a mulher a abortar variam de acordo com a sociedade e a cultura, no Brasil atualmente é considerado um caso de saúde pública, e nos faz atentar as seguintes situações que levam ao aborto:

- Restrições econômica: Para se dar uma vida digna a uma criança é necessária uma renda adequada e mulheres que não tem condições financeiras ou já tem outros filhos que sustenta com dificuldade concluem que não será viável uma nova gestação.

- Objetivos: Mulheres optam por interromper a gravidez para continuar seus planos e sonhos que teriam que ser adiados caso a gestação continuasse.

- A não aceitação familiar: Aquelas que estão iniciando a vida sexual sem a orientação necessária, pode ocorrer uma gravidez indesejada e interrompê-la é a melhor solução para evitar a rejeição e o abandono.

- Ausência de um parceiro: Não ter a presença de um pai influência na decisão da mulher em interromper a gestação, pois muitas sentem-se inseguras em não ter um apoio financeiro, afetivo e psicológico.

- Violência doméstica: Situações de violência em seu lar influenciam mulheres na questão do aborto, pois não conseguem levar a diante uma gravidez em um ambiente violento, onde não se tem poder de decisão e sofre agressões constantemente.

Alguns estudos revelam que mulheres que se autodeclaram pardas ou negras tiveram maior proporção nós abortos induzidos do que mulheres brancas, visto que está diretamente relacionada ao poder aquisitivo, uma vez que mulheres que dispõem de maior renda fazem abortos em clínicas particulares onde recebem melhor tratamento e não necessitam procurar ajuda, assim o procedimento fica encoberto não entrando nos números de aborto no país. Barbosa (2009)

\section{Categoria 1 - Consequências do aborto no entendimento da mulher.}

A norma técnica de Atenção humanizada ao abortamento, estabelece que as mulheres em processo de abortamento, espontâneo ou induzido, que procuram os serviços de saúde devem ser acolhidas, atendidas e tratadas com dignidade; e que a atenção tardia ao abortamento inseguro e às suas complicações pode ameaçar a vida, a saúde física e mental das mulheres (Brasil, 2005).

Segundo a Organização Mundial de Saúde, no ano de 2005, no Brasil foram realizados 1,4 milhão de abortos, e 42 a 50 milhões em todo o mundo.

No Brasil o aborto é um fenômeno frequente e persistente entre as mulheres de todas as classes sociais, grupos raciais, níveis educacionais e religiões: em 2016, quase 1 em cada 5 mulheres, aos 40 anos já realizou, pelo menos, um aborto. Em 2015, foram, aproximadamente, 416 mil mulheres (Diniz et al., 2017)

Um estudo realizado no ano de 1998, em 27 países, atestou as principais razões que levam as mulheres a um aborto induzido, são eles: $30,9 \%$ - eu não quero (mais) crianças, $21,1 \%$ - eu quero adiar a maternidade, $19,9 \%$ - ter um filho vai 
atrapalhar meus estudos ou trabalho, 6,6\% - eu não tenho condições financeiras agora, 4,4\% - tenho problema no meu relacionamento e meu parceiro não quer essa gravidez, 1,5\% - sou muito jovem ou meu pai ou outro(s) não querem minha gravidez, 9,8\% - minha saúde mental está em risco, 3,1\% - há um risco para a saúde do feto, 1,1 \% - minha saúde física está em risco, 1,6\% - outras razões apontadas. (Azevedo et al., 2018)

O abortamento espontâneo ocorre em aproximadamente $10 \%$ das gestações, envolvendo sentimento de perda, culpa pela impossibilidade de levar a gestação a termo, além de trazer complicações para o sistema reprodutivo, requerendo atenção técnica adequada, segura e humanizada (Brasil, 2005).

Sendo assim, o abortamento representa grave problema de saúde pública no Brasil, com sua discussão envolvendo complexo conjunto de aspectos legais, morais, religiosos, sociais e culturais.

O abortamento representa uma das principais causas de mortalidade materna no Brasil. Pesquisa realizada em 2003 por Laurenti et al., em todas as capitais e no Distrito Federal, permitiu evidenciar valor corrigido de 74 óbitos/100.000 nascidos vivos no País, diferentemente do que ocorre em países desenvolvidos, onde essas taxas de morte, especificamente por aborto, são reduzidas.

Além das consequências físicas decorrente de hemorragias e infecções, as mulheres também experimentam diversos sentimentos e sensações, como a dor, o arrependimento ou sensação de incapacidade por não ter sido capaz de leva a gestação até o fim, culpa, medo de punições legais, morais e religiosas, tristeza, entre outros.

O processo de abortamento para a maioria das mulheres é doloroso e desconfortável, seja pela utilização de métodos abortivos ou curetagem, quando feito por um profissional inadequado, um meio propício a complicações ou através de procedimentos radicais e sem acompanhamento esse processo pode se tornar ainda mais penoso e muitas vezes mortal.

Após realizarem o aborto, a maioria das mulheres relata um profundo sentimento de culpa, uma dor intimamente ligada a perda e ao sentimento de remorso ou incapacidade. Devido aos sentimentos que acompanham a perda, o medo de serem expostas e humilhadas, muitas mulheres preferem a solidão e o isolamento para lidar com a sua experiência. Sem apoio e com conflito interno devido a violação de regras morais, legais e religiosas, algumas mulheres entram em quadros depressivos.

\section{Categoria 2 - Atuação da enfermagem frente ao processo de morte vivenciado por mulheres que sofreram aborto.}

$\mathrm{O}$ aborto sempre foi visto pela sociedade apenas como uma forma monstruosa de tirar a vida de um inocente, ou a insuficiência da mulher em conseguir gerar uma vida, sendo assim, deixado de lado os fatores que levaram aquela mulher a devida situação que se encontra (seja induzido ou espontâneo). Não sendo diferente a forma como alguns profissionais de saúde se porta quando precisam prestar esse tipo de atendimento.

Ainda é muito comum ver mulheres sendo descriminadas e deixadas de lado esperando horas por atendimento como forma de punição pelo que aconteceu, já que o aborto é considerado um crime, ignorando seja qual for as circunstâncias que as levaram até ali, de imediato.

Segundo a Norma Técnica de Atenção Humanizada ao Abortamento, esta atividade atinge mulheres jovens, interferindo em sua saúde física, mental e reprodutiva, por causar complicações físicas imediatas, sendo feito de forma insegura em caso de ser induzido ou pela demora no atendimento por preconceito e desumanização dos profissionais de saúde que deixam suas questões religiosas ou morais interferirem erroneamente ao cuidado das mesmas (Brasil, 2005).

De acordo com Pitillin, cabe aos profissionais de saúde prestar uma assistência humanizada a mulher, mantendo a ética e o sigilo profissional, enquanto atende as suas reais necessidades sem menosprezar a dificuldade de cada uma. Pitilin et al. (2016).

O processo de abortamento é para muitas mulheres desconfortável e doloroso, necessitando de atenção médica 
adequada para o controle da dor e quadros de ansiedade, depressão e outras complicações. Situações emocionais influenciam na percepção da mulher e podem dificultar a assistência, exigindo do profissional de saúde atitudes amigáveis e tranquilizadoras. As instituições hospitalares devem estar equipadas e seus profissionais treinados para atuar nos casos de complicações, sendo o apoio verbal de suma importância para garantir um cuidado holístico.

A inadequação do controle da dor provoca nas mulheres sofrimentos desnecessários, além de aumentar suas ansiedades e de expor essas mulheres a mais complicações, provocando insatisfação quanto à qualidade da atenção prestada. Em todos os casos de atenção ao abortamento, na indução farmacológica ou nos outros procedimentos de esvaziamento (AMIU ou curetagem), avaliação clínica criteriosa, esclarecimentos sobre os procedimentos que serão utilizados, apoio afetuoso, empático e humanizado proporcionam ambiente favorável para abordagem menos medicamentosa, com menores riscos de complicações, menos custos, e mais rápida recuperação (Brasil, 2005).

Dentre as principais condutas de enfermagem estão na analgesia para controle da dor, no sangramento pós curetagem e na orientação da não manutenção das relações sexuais.

O enfermeiro, por ser quem tem o primeiro contato com a paciente e está presente durante todo o cuidado, pode também perceber o surgimento de quadros de ansiedade, melancolia, depressão, agitação constante, tendências suicidas, agressividade, entre outros comportamentos, e assim, sugerir que a paciente seja encaminhada ao serviço psicológico adequado para que tenha o acompanhamento necessário, o que fará com que a paciente se sinta mais acolhida e receptiva para maior eficácia do tratamento, de forma que a ajude a lidar com o desconforto causado após o ocorrido.

\section{Considerações Finais}

É possível perceber o quanto a interrupção da gestação é um evento traumático à mulher e representa riscos reais à saúde. Evidenciou-se que os artigos selecionados corroboram com a literatura, na qual aborto é elencado como uma das maiores causas de morte materna no Brasil (Brasil, 2005).

Além das complicações físicas, percebe-se que todos os tipos de aborto causam profundo sentimento de perda, arrependimento ou incapacidade na mulher que a vivência.

O medo do julgamento aliado com a falta de compreensão e apoio, fazem essas mulheres arcarem sozinhas com a dor. O isolamento recoberto pela autopunição, o receio de ser humilhada e exposta faz com que se torne mais propensa a distúrbios mentais como depressão, ansiedade e síndrome do pânico.

A necessidade da mulher por cuidados destinados as complicações do abortamento, sejam físicas ou psicológicas do processo, deixa inteligível a importância da assistência adequada, está então devendo ser aperfeiçoada através de educação em saúde continuada e treinamento adequado pelos profissionais de enfermagem.

Preservar a vida da mulher assim como a saúde mental é uma questão de saúde pública que dependerá diretamente do suporte e ajuda que iremos dispor, independente de questões morais, legais e religiosas, a assistência deverá ser integral e universal, visando os princípios da humanização.

A partir das pesquisas realizadas para a construção deste estudo, os autores notaram a necessidade de elaborar outras buscas sobre a temática e área envolvidas em questão, aprofundando-se cada vez mais devido a escassez de material existente. Desta maneira, acreditamos que futuramente teremos outros projetos para apresentar e assim, colaborar com a pesquisa, as comunidades acadêmicas e principalmente com a sociedade. 
Research, Society and Development, v. 10, n. 13, e36101320507, 2021

(CC BY 4.0) | ISSN 2525-3409 | DOI: http://dx.doi.org/10.33448/rsd-v10i13.20507

\section{Referências}

Alves, N. C. Triviños. A. N. S. (2013) Introdução à Pesquisa em Ciências Sociais: a Pesquisa Qualitativa em Educação-O Positivismo, A Fenomenologia, O Marxismo. Formação (Online), 1(20).

Azedevo, A. A., Ferreira, S. C., \& Faustino, T. W. (2018). Aborto: Legalizar ou não? Revista Científica Integrada, Guarujá.

Barbosa, R. M., Pinho, A. D. A., Santos, N. S., Filipe, E., Villela, W., \& Aidar, T. (2009). Aborto induzido entre mulheres em idade reprodutiva vivendo e não vivendo com HIV/aids no Brasil. Ciência \& Saúde Coletiva, 14, 1085-1099.

Bardin, L. (2016). Análise de conteúdo. Almedina Brasil.

Borsari, C. M. G., Nomura, R. M. Y., Benute, G. G., Nonnenmacher, D., Lucia, M. C. S. D., \& Francisco, R. P. V. (2012). O aborto inseguro é um problema de saúde pública. Femina.

Brasil. Ministério da Saúde. Área Técnica de Saúde da Mulher. Norma Técnica Atenção Humanizada ao Abortamento.

Brasil. Portal da Câmara dos deputados. Aborto é a quarta causa de mortalidade materna. Agência Câmara de Notícias.

Del Priore, M. (1993). Ao sul do corpo: condição feminina, maternidades e mentalidades no Brasil Colônia. Edunb.

Denzin, N. K., \& Lincoln, Y. S. (Eds.). (2011). The Sage handbook of qualitative research. sage.

Diniz, D., Medeiros, M., \& Madeiro, A. (2017). Pesquisa nacional de aborto 2016. Ciência \& Saúde Coletiva, 22, 653-660.

Flick, U. (2009). Desenho da pesquisa qualitativa-Coleção pesquisa qualitativa Editora Artmed.

Galeotti, G. (2007). História do Aborto. 102-104.

Pitilin, É. D. B., Banazeski, A. C., Bedin, R., \& Gasparin, V. A. (2016). Assistência de enfermagem em situações de aborto induzido/provocado: uma revisão integrativa da literatura. Enfermería global.

Souza, A. S. de (2007). Pesquisa qualitativa em administração: teoria e prática, RAC - Revista de Administração Contemporânea (Journal of Contemporary Administration), ANPAD - Associação Nacional de Pós-Graduação e Pesquisa em Administração, 11(2), $235-237$. 